\title{
THE STARTING SOLUTION FOR TWO-DIMENSIONAL HEAT CONDUCTION PROBLEMS WITH CHANGE OF PHASE*
}

\author{
BY \\ BRUNO A. BOLEY1 \\ (Columbia University) \\ AND \\ HARVEY P. YAGODA \\ (Stevens Institute of Technology)
}

\begin{abstract}
A short-time solution for two-dimensional change-of-phase problems for a half space is developed. The problems are such that melting starts at a point of the surface and spreads both along the surface and towards the interior of the body. Heating is applied by an analytic but otherwise general heat input.

It is found that the shape of the melt interface is, when normalized, a universal function, i.e. independent of both the applied heat input and the material properties. Initial melt propagation normal to and along the surface is proportional to $y^{3 / 2}$ and $y^{1 / 2}$ respectively, where $y$ is the nondimensional time measured from the start of melting.

1. Introduction. Numerous studies have been undertaken in the field of heat conduction including changes of phase since the early investigations of Lamé and Clapeyron [1], Neumann [2] and Stefan [3]; ${ }^{2}$ the large majority of these has been restricted however to one-dimensional problems, usually in rectangular coordinates, though cylindrical and spherical one-dimensional problems have also been considered. The present study constitutes what is believed to be the first exact short-time analytical solution of a truly two-dimensional problem; that is, one in which a spread of the melting interface occurs in two dimensions.

An analytic study of two-dimensional change-of-phase problems was previously carried out in [5], where such problems were classified according to the behavior of the liquid-solid interface. Referring, to be specific, to a body which starts to melt at some points of its surface, the problem was there said to be of Class I if the unmelted portion of the surface remained unchanged for all times. If, on the other hand, the melted portion of the surface was initially finite and not vanishing and increased with time, then the problem was categorized as of Class II. Finally, if melting started at a point and then spread along the surface (as well as into the interior), the problem was said to be of Class III. [5] presented a complete method of solution for problems of Class I; the development of a starting solution for problems of Class III is, as has been mentioned, the object of the present study.

The method of solution employed here makes use of the "embedding technique,"
\end{abstract}

\footnotetext{
* Received December 26, 1967; revised version received April 9, 1968. The work described here was supported by a grant of the Office of Naval Research, and formed part of a dissertation toward the Eng. Sc. D. degree at Columbia University.

${ }^{1}$ Present address: Cornell University.

${ }^{2}$ An interesting discussion of this early work, as well as extensions and indications of some important unsolved problems, was given by Brillouin [4].
} 
which was introduced for one-dimensional problems in [6], and employed in [5] for problems of Class I. In this approach, the melting or solidifying body of changing dimensions is mathematically extended to occupy the space filled by the actual body before change of phase. An unknown fictitious heat input is applied at the boundary of the extended body, whose magnitude is then determined in such a manner as to satisfy the actual boundary conditions on the moving liquid-solid interface. The principal advantage of this technique is that it allows an explicit expression for the temperature to be written in terms of the fictitious heat input. A general one-dimensional starting solution was constructed by this method in [9].

The solution presented applies equally to the melting of an initially solid body and to the solidification of an initially liquid mass; for simplicity therefore only the former of these possibilities will in general be discussed.

Problems of melting may be classified according to the behavior of the liquid phase, that is, depending on whether the liquid is allowed to remain stationary upon formation, is ablated in a prescribed manner or is instantaneously removed. The latter case will be considered here, but it should be remembered that in one-dimensional problems the starting solution is independent of the rate of ablation [11], including the limiting cases of zero rate (stationary liquid) and infinite rate (instantaneous removal). It may be supposed on physical grounds that a similar statement can be made for two-dimensional problems: if that is the case, the present solution can be taken to apply regardless of the behavior of the liquid.

The solution presented here is restricted to short times after the start of melting; it allows for arbitrary heating conditions prior to that time, but is restricted to heat inputs which vary smoothly in the neighborhood of the time of melt initiation. An analytic solution valid for somewhat longer values of the time could be obtained by the technique here employed, but for longer times yet the present technique would probably be more conveniently employed in conjunction with a numerical analysis.

The starting solution, such as the one developed here, is interesting because it can provide some basic insight into the early stages of the solidification or melting process. It is however useful also in other respects: it has been conveniently employed as a start of a numerical solution (e.g. [6]), or as a start of an approximate analytical solution (e.g. [12]) or as a check on the accuracy of approximate solutions (e.g. [13]). Furthermore, it is hoped that the work presented here will eventually lead to a more accurate though macroscopic study of the process of nucleation and of the early growth and morphology of dendrites and cellular clusters. The work which has been done in this area ${ }^{4}$ has considered two-dimensional heat conduction under several simplifying assumptions; significant among these was the assumption of the shape of the growth, while the determination of such a shape is one of the principal aims of the present approach. A further discussion of this aspect of the problem will be found in the last section of the paper.

The problem under consideration is formulated in Sec. 2 . Its solution is formally carried out in Sec. 3, where it is shown that all the conditions of the problem are satisfied on the basis of certain assumptions, a uniqueness theorem insuring that the only solution has thus been found. The actual solution is then obtained in Sec. 4; the results are summarized in Sec. 5 and discussed in Sec. 6.

${ }^{3}$ For a further discussion see [7]; this and [8] contain comprehensive bibliographies.

[14] - [17], for example; [4] may be consulted for an exhaustive bibliography. 
2. Formulation of the problem. Consider the half space $z>0$, initially solid, to be heated on the boundary $z=0$ by a heat input $Q(x, t)$, giving rise to a temperature $T(x, z, t)$. Assume that at a time $t=t_{m}$, the temperature reaches for the first time the melting temperature $T_{m}$ of the material, and that it does so at a point on the boundary ${ }^{5}$ which may be taken without loss of generality as the origin; thus

$$
T\left(0,0, t_{m}\right)=T_{m} .
$$

The determination of the temperature for $t \leq t_{m}$ is a straightforward problem in the theory of heat conduction and need not concern us here; it will be found useful to recall, however, that it can be written in terms of $Q$ and of the initial temperature $T_{I}(x, z)$ at $t=t_{0}$ as [18], [19]:

$$
\begin{aligned}
T(x, z, t)= & \frac{\kappa}{k} \int_{t_{0}}^{t} \int_{-\infty}^{\infty} Q\left(x_{1}, \tau\right) \frac{\exp \left[-\left(\left(x-x_{1}\right)^{2}+z^{2}\right) /(4 \kappa(t-\tau))\right]}{2 \pi \kappa(t-\tau)} d x_{1} d \tau \\
& +\int_{0}^{\infty} \int_{-\infty}^{\infty} T_{I}\left(x_{1}, z_{1}\right) \frac{\exp \left[-\left(x-x_{1}\right)^{2} / 4 \kappa\left(t-t_{0}\right)\right]}{4 \pi \kappa\left(t-t_{0}\right)} \\
& \cdot\left[\exp \left[-\left(z-z_{1}\right)^{2} / 4 \kappa\left(t-t_{0}\right)\right]+\exp \left[-\left(z+z_{1}\right)^{2} / 4 \kappa\left(t-t_{0}\right)\right]\right] d x_{1} d z_{1} .
\end{aligned}
$$

The heat input $Q(x, t)$ continues to be applied after $t_{m}$, and the body continues to melt, so that, for any $t>t_{m}$, the solid will occupy a region smaller than the original half space and will in fact be bounded by the curve (Fig. 1a):

$$
z= \begin{cases}s(x, t), & |x| \leq x_{0}(t) \\ 0, & |x| \geq x_{0}(t)\end{cases}
$$

where the unknown quantities $s$ and $x_{0}$ satisfy the condition

$$
s\left(x_{0}, t\right)=0, \quad x_{0}\left(t_{m}\right)=0
$$

and where it has been assumed (as will be done for simplicity in the remainder of this paper) that the problem is symmetrical about $x=0$. The liquid, which would occupy

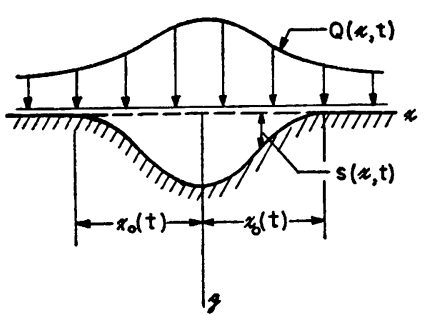

(a) REPRESENTATION OF THE PROBLEM (Q opplied on $z=s$ )

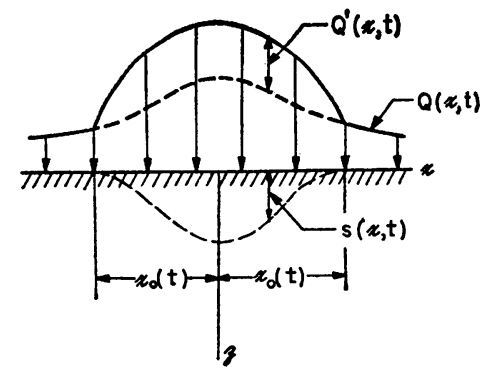

(b) REPRESENTATION OF THE EXTENDED PROBLEM

FIG. 1

${ }^{5}$ The time $t_{m}$ is the time of start of melting, criteria for which are given in [5], [9]. 
the region $0<z<s(x, t),|x|<x_{0}(t)$, is assumed here to be instantaneously removed upon formation.

The mathematical formulation of the problem after the start of melting consists of the Fourier heat conduction equation:

$$
\kappa\left(\partial^{2} T / \partial x^{2}+\partial^{2} T / \partial z^{2}\right)=\partial T / \partial t,
$$

the initial condition:

$$
T=T\left(x, z, t_{m}\right) \quad \text { at } \quad t=t_{m}
$$

and the moving-boundary conditions: ${ }^{6}$

$$
\begin{gathered}
T(x, s, t)=T_{m} \quad|x| \leq x_{0}(t), \\
-k \frac{\partial T}{\partial z}=Q(x, t) \quad \text { for } \quad|x| \geq x_{0}(t) \quad \text { on } z=0, \\
-k\left[1+\left(\frac{\partial s}{\partial x}\right)^{2}\right] \frac{\partial T}{\partial z}=Q(x, t)-\rho l \frac{\partial s}{\partial t} \quad \text { for } \quad|x| \leq x_{0}(t) \quad \text { on } z=s(x, t),
\end{gathered}
$$

as well as conditions (3a). Note that the right-hand side of Eq. (5) is a known function, obtained from the premelting solution. The conductivity $k$, the diffusivity $\kappa$, the density $\rho$, and the latent heat $l$ are taken to be constants.

It can be shown that the solution to the above boundary-value problem is unique. Indeed, it was proved in [20] that not more than one solution exists if $Q(x, t)$ and its integral over the moving portion of the boundary are prescribed; that proof is readily extended to the case in which (as in the present problem) $Q$ and the total heat input over the entire boundary are prescribed.

3. Formulation in terms of the embedding technique. To obtain a solution to the problem formulated in the preceding section, we extend mathematically the temperature so as to be defined in the entire half space $z>0$, and to satisfy throughout that region Eq. (4) and, at $t=t_{m}$, Eq. (5). The boundary condition on $z=0$ is already prescribed by Eq. (7a) for the region $|x|>x_{0}$, while for $|x|<x_{0}$ it may be arbitrarily prescribed, since the boundary is there fictitious. It is convenient to introduce a fictitious heat input $Q^{\prime}(x, t)$ to be applied over this fictitious portion of the boundary, and to express the boundary condition as

$$
-k(\partial T / \partial z)=Q^{*}(x, t)+Q^{\prime}(x, t) \quad t>t_{m},|x|<x_{0}(t)
$$

where $Q^{*}(x, t)$ is the analytic continuation of the premelting heat input $Q$. A representation of the extended problem is shown in Fig. $1 \mathrm{~b}$.

The extended temperature can now be immediately expressed by means of Eq. (2) as

$$
T(x, z, t)=T^{*}(x, z, t)+T^{\prime}(x, z, t),
$$

where

$$
T^{\prime}(x, z, t)=\frac{\kappa}{k} \int_{t_{m}}^{t} \int_{-x_{0}(\tau)}^{x_{0}(\tau)} Q^{\prime}\left(x_{1}, \tau\right) \frac{\exp \left[-\left(\left(x-x_{1}\right)^{2}+z^{2}\right) /(4 \kappa(t-\tau))\right]}{2 \pi \kappa(t-\tau)} d x_{1} d \tau
$$

${ }^{6} \mathrm{~A}$ derivation of the heat flux interface condition (7b) can be found in [5]; it assumes that the resultant of the heat flux vector is in the $z$-direction. 
and therefore (since $Q^{\prime}$ has been applied only in $|x|<x_{0}$ )

$$
\partial T^{\prime} / \partial z=0 \quad \text { on } z=0,|x|>x_{0} .
$$

Note that the temperature $T$ automatically satisfies Eq. (7a), in view of (9b), and because the temperature $T^{*}$ is that caused by the heat input $Q^{*}$ which is here taken to be equal to $Q$ for $|x|>x_{0} . T^{*}$ is therefore the analytic continuation of the premelting temperature given by Eq. (2) and can thus be expressed in terms of the temperature and its derivatives at $t_{m}$ as

$$
\begin{aligned}
\frac{T^{*}}{T_{m}}(X, Z, y)= & 1+y \frac{\partial\left(\frac{T^{*}}{T_{m}}\right)}{\partial y}+\frac{y^{2}}{2 !} \frac{\partial^{2}\left(\frac{T^{*}}{T_{m}}\right)}{\partial y^{2}}+\cdots \\
& +Z\left[\frac{\partial\left(\frac{T^{*}}{T_{m}}\right)}{\partial Z}+y \frac{\partial^{2}\left(\frac{T^{*}}{T_{m}}\right)}{\partial Z \partial y}+\cdots\right]+\frac{Z^{2}}{2 !}\left[\frac{\partial^{2}\left(\frac{T^{*}}{T_{m}}\right)}{\partial Z^{2}}+\cdots\right]+\cdots \\
& +\frac{X^{2}}{2 !}\left[\frac{\partial^{2}\left(\frac{T^{*}}{T_{m}}\right)}{\partial X^{2}}+y \frac{\partial^{3}\left(\frac{T^{*}}{T_{m}}\right)}{\partial X^{2} \partial y}+\cdots+Z \frac{\partial^{3}\left(\frac{T^{*}}{T_{m}}\right)}{\partial X^{2} \partial Z}+\cdots\right] \\
& +\frac{X^{4}}{4 !}\left[\frac{\partial^{4}\left(\frac{T^{*}}{T_{m}}\right)}{\partial X^{4}}+y \frac{\partial^{5}\left(\frac{T^{*}}{T_{m}}\right)}{\partial X^{4} \partial y}+\cdots+Z \frac{\partial^{5}\left(\frac{T^{*}}{T_{m}}\right)}{\partial X^{4} \partial Z}+\cdots\right]+\cdots
\end{aligned}
$$

where all derivatives of $T^{*}$ must be evaluated at $X=Z=y=0$. The dimensionless variables

$$
y=\left(t-t_{m}\right) / t_{m} \quad X=x / 2\left(\kappa t_{m}\right)^{1 / 2} \quad Z=z / 2\left(\kappa t_{m}\right)^{1 / 2}
$$

have been used; with the further dimensionless notation ${ }^{7}$

$$
X_{0}(y)=\frac{x_{0}}{2\left(\kappa t_{m}\right)^{1 / 2}}, \quad \xi=\frac{s}{2\left(\kappa t_{m}\right)^{1 / 2}}, \quad Q_{0}=\frac{k T_{m}}{2}\left(\frac{\pi}{\kappa t_{m}}\right)^{1 / 2}, \quad m=\frac{\pi^{1 / 2} c T_{m}}{2 l}=\frac{Q_{0}}{\rho l}\left(\frac{t_{m}}{\kappa}\right)^{1 / 2}
$$

the temperature (9) assumes the form

$$
\begin{aligned}
& \frac{T}{T_{m}}(X, Z, y)=\frac{T^{*}}{T_{m}}(X, Z, y) \\
& \quad+\int_{0}^{y} \frac{e^{-Z^{2 / y_{1}}}}{2 \pi^{1 / 2} y_{1}} \int_{-X_{0}\left(y-y_{1}\right)}^{X_{0}\left(y-y_{1}\right)} \frac{Q^{\prime}\left(X_{1}, y-y_{1}\right)}{Q_{0}} \exp \left[-\left(X-X_{1}\right)^{2} / y_{1}\right] d X_{1} d y_{1} .
\end{aligned}
$$

Equation (12) gives the temperature throughout the body after melting in terms of the three unknown functions $\xi(X, y), Q^{\prime}(X, y)$ and $X_{0}(y)$. These unknowns will be found from the interface conditions (6) and (7b), as well as another condition to be discussed later (i.e. Eq. (39)].

Equations (6) and (7b), when put in dimensionless form, become respectively

'The reference heat input $Q_{0}$ has the following physical meaning: if a constant heat input of magnitude $Q_{0}$ is applied uniformly along $x$ on the free surface of a half space, then the relation between the melting time $t_{m}$ and $Q_{0}$ is that given by the third of Eqs. (11b). 


$$
\begin{gathered}
\int_{0}^{\nu} \frac{e^{-\xi^{2} / y_{1}}}{2 \pi^{1 / 2} y_{1}} \int_{-X_{0}\left(y-y_{1}\right)}^{X_{0}\left(\nu-y_{1}\right)} \frac{Q^{\prime}\left(X_{1}, y-y_{1}\right)}{Q_{0}} \exp \left[-\left(X-X_{1}\right)^{2} / y_{1}\right] d X_{1} d y_{1}=1-\frac{T^{*}}{T_{m}}(X, \xi, y), \\
-\frac{1}{\pi^{1 / 2}}\left[1+\left(\frac{\partial \xi}{\partial X}\right)^{2}\right]\left[\frac{\partial\left(\frac{T^{*}}{T_{m}}\right)}{\partial Z}-\xi \int_{0}^{\nu} \frac{e^{-\xi^{2} / y_{1}}}{\pi^{1 / 2} y_{1}^{2}} \int_{-X_{0}\left(\nu-y_{1}\right)}^{X_{0}\left(\nu-y_{1}\right)} \frac{Q^{\prime}\left(X_{1}, y-y_{1}\right)}{Q_{0}}\right. \\
\left.\cdot \exp \left[-\left(X-X_{1}\right)^{2} / y_{1}\right] d X_{1} d y_{1}\right]=\frac{Q(X, y)}{Q_{0}}-\frac{2}{m} \frac{\partial \xi}{\partial y}(X, y)
\end{gathered}
$$

for $|X| \leq X_{0}(y)$, on $Z=\xi(X, y)$.

These two integro-differential equations will now be solved under the assumption that

$$
\frac{Q^{\prime}(X, y)}{Q_{0}}=\sum_{i=0}^{\infty} 4^{i} F_{i}(y) X^{2 i}, \quad|X|<X_{0}(y) .
$$

Substitution of this expression into (12) gives the temperature as

$$
\frac{T}{T_{m}}(X, Z, y)=\frac{T^{*}}{T_{m}}(X, Z, y)+\sum_{i=0}^{\infty} \int_{0}^{\nu} \frac{4^{i} F_{i}\left(y-y_{1}\right)}{2 \pi^{1 / 2} y_{1}} e^{-Z^{*} / \nu_{1}} I_{(2 i)} d y_{1}
$$

where

$$
\begin{aligned}
I_{j}\left(X, X_{0}, y_{1}\right) & \equiv I_{j}\left[X, X_{0}\left(y-y_{1}\right), y_{1}\right] \\
& =\int_{-X_{0}\left(\nu-y_{1}\right)}^{X_{0}\left(\nu-y_{1}\right)} X_{1}^{j} \exp \left[-\left(X-X_{1}\right)^{2} / y_{1}\right] d X_{1} .
\end{aligned}
$$

Expression (15a) gives the temperature at all points of the body.

(a) Consideration of the Interface Condition $T=T_{m}$, Eqs. (6) or (13a). Expand the function $I_{(2 i)}$ in (15a) in powers of $X$, only even powers appearing because of symmetry, and use the result to rewrite Eq. (13a) as

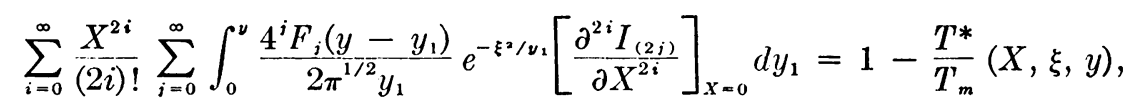

where the functions $I_{(2 i)}$ and their even derivatives can be expressed as

$$
\left[\frac{\partial^{2 i} I_{(2 j)}}{\partial X^{2 i}}\right]_{X=0}=y_{1}^{i-i+1 / 2} J_{i j}\left(\frac{X_{0}}{y_{1}^{1 / 2}}\right)
$$

where

$$
\begin{aligned}
& J_{i i}\left(\frac{X_{0}}{y_{1}^{1 / 2}}\right)=4^{i} \pi^{1 / 2} D_{i+1, i+1} \operatorname{erf}\left(\frac{X_{0}}{y_{1}^{1 / 2}}\right) \\
& -\sum_{n=1}^{i+1}\left(1-\delta_{i+i, n-1}\right) \gamma_{n}(i) \sum_{k=0}^{i+i-n} \frac{(2 i+2 j-2 n+1) ! !}{2^{k}(2 i+2 j-2 n-2 k+1) ! !}\left(\frac{X_{0}}{y_{1}^{1 / 2}}\right)^{2(i+j-n-k)+1} e^{-X_{0} * / v_{1}}
\end{aligned}
$$

and

$$
D_{i+1, j+1}=4^{-i} \sum_{n=1}^{i+1} \gamma_{n}(i) \frac{(2 i+2 j-2 n+3) ! !}{(2 i+2 j-2 n+3) 2^{i+j-n+1}}
$$


and where

$$
\gamma_{n}(i)=\frac{(-1)^{n-1} 4^{i-n+1}(2 i) !}{(n-1) !(2 i-2 n+2) !}
$$

are the coefficients appearing in the even Hermite polynomials [21]

$$
H_{2 i}(x)=\sum_{n=1}^{i+1} \gamma_{n}(i) x^{2(i-n+1)}
$$

Note that $J_{i j}$ contains no error function term when $j<i$ since $D_{i j}=0$ for these cases, demonstration of which is given in Appendix B.

Equation (17a) has been derived with the aid of successive integrations by parts of the integrals

$$
\begin{aligned}
& \left.\frac{\partial^{2 i} I_{(2 j)}}{\partial X^{2 i}}\right]_{X=0}=\int_{-X_{0}\left(y-y_{1}\right)}^{X_{0}\left(y-y_{1}\right)} X_{1}^{2 j}\left[\frac{\partial^{2 i}}{\partial X^{2 i}} \exp -\left[\left(X-X_{1}\right)^{2} / y_{1}\right]\right]_{X=0} d X_{1}, \quad i, j \geq 0 \\
& =y_{1}^{-i} \int_{-X_{0}\left(y-y_{1}\right)}^{X_{0}\left(y-y_{1}\right)} X_{1}^{2 i} H_{2 i}\left(\frac{X_{1}}{y_{1}^{1 / 2}}\right) e^{-X_{1}^{2} / y_{1}} d X_{1}
\end{aligned}
$$

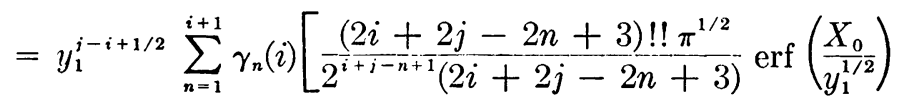

$$
\begin{aligned}
& \left.-\left(1-\delta_{i+j, n-1}\right) \sum_{k=0}^{i+j-n} \frac{(2 i+2 j-2 n+1) ! !}{2^{k}(2 i+2 j-2 n-2 k+1) ! !}\left(\frac{X_{0}}{y_{1}^{1 / 2}}\right)^{2(i+j-n-k)+1} e^{-X_{0}: / y_{2}}\right]
\end{aligned}
$$

where $u=X_{1} / y_{1}^{1 / 2}$.

Primary interest is centered here on developing a starting solution, for which only the lowest-order terms on the right-hand side of Eq. (16), expanded by means of (10), need be kept. If this is done, and if coefficients of like powers of $X$ on both sides of the equation are matched, the following infinite set of simultaneous integral equations for the functions $F_{i}$ is obtained:

$$
\begin{aligned}
\sum_{j=0}^{\infty} \int_{0}^{\nu} \frac{4^{j} F_{i}\left(y-y_{1}\right)}{2 \pi^{1 / 2} y_{1}} e^{-\xi^{2} / \nu_{1}}\left[\frac{\partial^{2 i} I_{(2 j)}}{\partial X^{2 i}}\right]_{X=0} d y_{1} \\
=\left\{\begin{array}{l}
-y\left[\frac{\partial\left(\frac{T^{*}}{T_{m}}\right)}{\partial y}\right]_{0,0,0}, \quad i=0 \\
{\left[\frac{\partial^{2 i}\left(\frac{T^{*}}{T_{m}}\right)}{\partial X^{2 i}}\right]_{0,0,0}, \quad i=1,2, \ldots}
\end{array}, y \ll 1\right.
\end{aligned}
$$

The first three of these equations are given explicitly in Appendix A.

It is now necessary to reduce the left-hand side of this equation to an expression valid for small values of $y$. It is shown in [9], [6], [23] that, for $y \ll 1$, the exponential $e^{-\xi^{2} / \nu_{1}}$ may be replaced by unity, provided that

$$
\lim _{y \rightarrow 0} \frac{\xi(X, y)}{y^{1 / 2}}=0
$$

and that

$$
\left|1-F_{i}\left(y-y_{1}\right) / F_{i}(y)\right| \ll 1 \text { for } y_{1} \rightarrow 0 \text {. }
$$


It will be assumed here, and verified a posteriori, that (21) holds. Thus, using (17), the first of Eqs. (20), i.e. for $i=0$, becomes ${ }^{8}$

$$
\sum_{i=0}^{\infty} \int_{0}^{\nu} \frac{4^{j} F_{i}\left(y-y_{1}\right)}{2 \pi^{1 / 2}} y_{1}^{j-1 / 2} J_{0 i}\left(\frac{X_{0}}{y_{1}^{1 / 2}}\right) d y_{1}=-y\left[\frac{\partial\left(\frac{T^{*}}{T_{m}}\right)}{\partial y}\right]_{0,0,0}, \quad y \ll 1,
$$

Clearly the left-hand side will be of the correct form (i.e. proportional to $y$ ) if

$$
F_{j}(y)=A_{i+1} / y^{i-1 / 2} \text { and } X_{0}(y)=A y^{1 / 2}
$$

where $A_{i}$ and $A$ are constants, since it then reduces to

$$
y \sum_{i=0}^{\infty} \frac{4^{i}}{2 \pi^{1 / 2}} A_{j+1} \int_{0}^{1}\left(\frac{Y}{1-Y}\right)^{j-1 / 2} J_{0 i}\left(A\left(\frac{1-Y}{Y}\right)^{1 / 2}\right) d Y
$$

where

$$
Y=y_{1} / y \text {. }
$$

Assumption (24) will represent a solution of the problem only if the constants $A_{i}$ can be adjusted so as to satisfy all of Eqs. (20), for short times, while the constant $A$ will be chosen later so as to satisfy the required conditions of continuity. The function $\xi$ will be found by use of the heat-input boundary condition (13b). All equations of the problem are then satisfied, and the uniqueness theorem insures that this is indeed the solution.

Consider first Eqs. (20). Substitution of (24) into (20) and use of transformation (26) shows, in a manner analogous to that just employed for $i=0$, that only integrals of the type (25) arise, proportional however to $y^{0}, y^{-1}, y^{-2}, \cdots$ for $i=1,2,3, \cdots$. The first of these powers is the same as that required by the right-hand side of Eq. (20), while the others are smaller; hence the right-hand sides for $i=0,1$ must be taken from (20), while for $i \geq 2$ they must be set equal to zero. The constants $A_{i}$ are therefore obtained from the infinite set of linear algebraic equations

$$
\sum_{i=1}^{\infty} I_{i j}(.1) A_{i}=(-1)^{i} C_{i}, \quad i=1,2,3, \cdots
$$

where

$$
\begin{gathered}
\left.\left.C_{1}=\frac{\partial\left(\frac{T^{*}}{T_{m}}\right)}{\partial y}\right]_{0,0,0}>0 ; \quad C_{2}=-\frac{\partial^{2}\left(\frac{T^{*}}{T_{m}^{*}}\right)}{\partial X^{2}}\right]_{0,0,0}>0 \\
C_{i}=0, \quad i>2
\end{gathered}
$$

and where

$$
I_{i j}=\frac{4^{j-1}}{2 \pi^{1 / 2}} \int_{0}^{1} \frac{Y^{i-i-1 / 2}}{(1-Y)^{i-\overline{3} / \overline{2}}} J_{i-1, j-1}\left(A\left(\frac{1-Y}{Y}\right)^{1 / 2}\right) d Y .
$$

This completes the formal consideration of the first interface boundary condition, and we proceed to an examination of the second interface condition. It is clear, however,

${ }^{8}$ Recall that $X_{0}=X_{0}\left(y-y_{1}\right)$. 
that solution of Eqs. (27) presents a difficult problem because of the complicated dependence of the coefficients $I_{i j}$ on the parameter $A$. The solution of these equations, alternate expressions for $I_{i i}$, and the determination of $A$ are discussed in Sec. 4.

(b) Consideration of the Heat-Balance Interface Condition, Eqs. (7b) or (13b). We now turn to the heat-input boundary condition, Eq. (13b); with the aid of (14) and (15b), and expansion of the functions $I_{(2 i)}$, it can be rewritten as

$$
\begin{aligned}
\frac{2}{m} \frac{\partial \xi}{\partial y}(X, y)-\frac{Q(X, y)}{Q_{0}}=\frac{1}{\pi^{1 / 2}}\left[1+\left(\frac{\partial \xi}{\partial X}\right)^{2}\right] \\
\qquad\left\{\frac{\partial\left(\frac{T^{*}}{T_{m}}\right)}{\partial Z}-\frac{\xi}{\pi^{1 / 2}} \sum_{i=0}^{\infty} \frac{X^{2 i}}{(2 i) !} \int_{0}^{y} \sum_{j=0}^{\infty} \frac{4^{i} F_{j}\left(y-y_{1}\right)}{y_{1}^{2}} e^{\left.-\xi^{2 / y_{2}}\left[\frac{\partial^{2 i} I_{(2 i)}}{\partial X^{2 i}}\right]_{X=0} d y_{1}\right\} .}\right.
\end{aligned}
$$

Substitution of (17) and (24) results in the following integrals whose evaluation to firstorder terms is shown in [23], under condition (21), to be

$$
\xi \int_{0}^{\nu} \sum_{i=0}^{\infty} 4^{i} A_{i+1} \frac{y_{1}^{i-i-3 / 2}}{\left(y-y_{1}\right)^{j-1 / 2}} e^{-\xi^{2} / \nu_{1}} J_{i i}\left(A\left(\frac{y-y_{1}}{y_{1}}\right)^{1 / 2}\right) d y_{1}=4^{i}(2 i) ! \pi \frac{A_{i+1}}{y^{i-1 / 2}} .
$$

Thus Eq. (29) finally becomes

$$
\frac{2}{m} \frac{\partial \xi}{\partial y}(X, y)=\frac{Q(X, y)-Q^{*}(X, 0)}{Q_{0}}-\sum_{i=0}^{\infty} 4^{i} A_{i+1} \frac{X^{2 i}}{y^{i-1 / 2}}, \quad y \ll 1 .
$$

Note that

$$
\frac{Q^{\prime}(X, y)}{Q_{0}}=\sum_{i=0}^{\infty} 4^{i} A_{i+1} \frac{X^{2 i}}{y^{i-1 / 2}} .
$$

With the condition $\xi(X, 0)=0$, Eq. (31) can be integrated to obtain $\xi(X, y)$. If

$$
\lim _{y \rightarrow 0} \frac{1}{y^{1 / 2}}\left|\frac{Q(X, y)-Q^{*}(X, 0)}{Q_{0}}\right|=0
$$

as assumed here, the melt depth is

$$
\xi(X, y)=-m \sum_{i=0}^{\infty} \frac{4^{i}}{3-2 i} A_{i+1} \frac{X^{2 i}}{y^{i-3 / 2}}
$$

or

$$
\xi(X, y)=-\frac{m}{4} y^{3 / 2} \sum_{i=1}^{\infty} \frac{4^{i}}{5-2 i} A_{i} A^{2(i-1)}\left(\frac{X}{X_{0}}\right)^{2(i-1)} .
$$

Equation (34b) gives the form of the melting interface, in terms of the coefficient $A$, which appears both explicitly and implicitly in the coefficients $A_{i}(A)$; the latter are assumed to have been found from Eqs. (27) in the manner discussed in the following section.

This completes the formal consideration of the heat-balance interface condition; it only remains to establish the equation for the determination of the parameter $A$, which will now be examined.

It will first be proved that the condition

$$
\xi\left(X_{0}, y\right) \equiv 0
$$


is already identically satisfied. Indeed the temperatures $T\left[X_{0}^{-}, \xi\left(X_{0}^{-}, y\right), y\right], T\left[X_{0}^{+}, 0, y\right]$ and $T\left[X_{0}^{+}, \xi\left(X_{0}^{-}, y\right), y\right]$ are all equal to $T_{m}$ : the first because it was set equal to $T_{m}$ in Eq. (16); the second because it happens that the expression obtained by setting $z=0$ in Eq. (15a) is identical with that of Eq. (23), resulting from (16) after use of the short time approximation (21) and since $I_{i}$ is a continuous function of $X$; the third for continuity ${ }^{9}$ of temperature across $X=X_{0}$ at $Z=\xi\left(X_{0}^{-}, y\right)$. The last temperature listed is, however, also given by

$$
T_{m}-\frac{Q\left(X_{0}, y\right)}{k} \xi\left(X_{0}^{-}, y\right) ;
$$

hence Eq. (35) holds. In other words, it has now been proved that Eqs. (27) and (35) cannot be independent; it will be indeed verified in the next section that this is the case (cf. Eq. 46). In other words, condition (35) which can be rewritten as

$$
\sum_{i=1}^{\infty} \frac{4^{i}}{5-2 i} A_{i} A^{2(i-1)}=0
$$

will also result from Eqs. (27)

The temperature $T\left[X_{0}^{-}, 0, y\right]$ is, however, also equal to $T_{m}$ by the same reasoning used in conjunction with $T\left[X_{0}^{+}, 0, y\right]$; hence, it is necessary that

$$
\frac{\partial T}{\partial z}\left(X_{0}^{-}, 0, y\right)=\frac{\partial T}{\partial z}\left(X_{0}^{+}, 0, y\right)=-\frac{Q\left(X_{0}, y\right)}{k} .
$$

Substituting into Eq. (7b), and omitting the term $(\partial s / \partial x)^{2}$ in accordance with the development of Eq. (30), we obtain the desired equation for the determination of $X_{0}(y)$ as

$$
\frac{\partial \xi}{\partial y}=0 \quad \text { at } \quad X=X_{0}(y) .
$$

This of course implies that

$$
Q^{\prime}\left[X_{0}(y), y\right]=0
$$

and can be written explicitly from Eq. (31) or (34) as

$$
\sum_{i=1}^{\infty} 4^{i-1} A_{i} A^{2(i-1)}=0 .
$$

The determination of the constants $A_{i}$ and $A$ must now be effected from Eqs. (27) and (40); this is done in Sec. 4.

Note that the total derivative of $\xi\left[X_{0}(y), y\right]$ with respect to $y$ is zero or

$$
\frac{\partial \xi}{\partial y}+\frac{\partial \xi}{\partial X} \frac{d X_{0}}{\partial y}=0 \quad \text { at } \quad X=X_{0}(y) .
$$

With (39), this implies that

$$
\partial \xi / \partial X=0 \quad \text { at } \quad X=X_{0}(y) .
$$

The quantity $\partial Q^{\prime} / \partial X$ is not necessarily zero at $X=X_{0}(y)$.

4. Solution. In order to complete the solution of the problem, it is now necessary to determine the constants $A_{i}$ and $A$ from Eqs. (27) and (40). Once these constants are

\footnotetext{
${ }^{9}$ Continuity in the interior of the body is assured in the solution by use of the Duhamel formula (9a) because of the lack of internal heat sources; even a discontinuous surface heat input will not result in interior temperature discontinuities [22].
} 
TABLE I

Coefficients $I_{i j}$ for $i, j=1,2, \ldots, 5$

\begin{tabular}{|c|c|c|c|c|c|}
\hline $11^{\prime}$ & 1 & 2 & 3 & 4 & 5 \\
\hline 1 & $\begin{array}{l}\frac{\pi}{4}-\frac{1}{\pi}[I(A)- \\
\left.\frac{1}{2} A e^{A^{2}} E i\left(A^{2}\right)\right]\end{array}$ & $\begin{array}{l}\frac{\pi}{2}+\frac{2}{\sqrt{x}}[-A-I(A)+ \\
\left.\left(A^{3}-\frac{A}{2}\right) e^{A^{2}} E I\left(A^{2}\right)\right]\end{array}$ & $\begin{array}{l}-9 \pi+\frac{2}{5 \pi}\left[-4 A^{3}+18 A+\right. \\
18 I(A)+\left(4 A^{5}-6 A^{3}+\right. \\
\left.9 A) e^{A^{2}} E 1\left(A^{2}\right)\right]\end{array}$ & $\begin{array}{l}150 \pi+\frac{8}{\pi}\left[-4 A^{5}+\frac{50}{3} A^{3}-75 A-\right. \\
75 I(A)+\left(4 A^{7}-10 A^{5}+25 A^{3}-\right. \\
\left.\left.\frac{75}{2} A\right) e^{A^{2}} E i\left(A^{2}\right)\right]\end{array}$ & $\begin{array}{l}-2940 \pi+\frac{112}{7 \pi}\left[-\frac{8}{7} A^{7}+\frac{28}{5} A^{5}-\frac{70}{3} A^{3}\right. \\
+105 A+105 I(A)+\left(\frac{8}{7} A^{9}-4 A^{7}+\right. \\
\left.\left.14 A^{5}-35 A^{3}+\frac{105}{2} A\right) e^{A^{2}} E 1\left(A^{2}\right)\right]\end{array} \mid$ \\
\hline 2 & $\begin{array}{l}-\frac{2}{\sqrt{\pi}} A^{-1}[1- \\
\left.A^{2} e^{A^{2}} E 1\left(A^{2}\right)\right]\end{array}$ & $\begin{array}{l}4 \pi-\frac{8}{T \pi}[A+2 I(A)- \\
\left.\left(A^{3}-A\right) e^{A^{2}} E i\left(A^{2}\right)\right]\end{array}$ & $\begin{array}{l}-48 \pi+\frac{32}{\sqrt{\pi}}\left[-A^{3}+6 A+\right. \\
6 I(A)+\left(A^{5}-2 A^{3}+\right. \\
\left.3 A) e^{A^{2}} E I\left(A^{2}\right)\right]\end{array}$ & $\begin{array}{l}720 \pi+\frac{64}{\pi}\left[-2 A^{5}+10 A^{3}-45 A-\right. \\
45 I(A)+\left(2 A^{7}-6 A^{5}+15 A^{3}-\right. \\
\left.\left.\frac{45}{2} A\right) e^{A^{2}} E i\left(A^{2}\right)\right]\end{array}$ & $\begin{array}{l}-13440 \pi+\frac{512}{\pi}\left[-A^{7}+\frac{28}{5} A^{5}-\frac{70}{3} A^{3}\right. \\
+105 A+105 I(A)+\left(A^{9}-4 A^{7}+\right. \\
\left.\left.14 A^{5}-35 A^{3}+\frac{105}{2} A\right) e^{A^{2}} E i\left(A^{2}\right)\right]\end{array}$ \\
\hline 3 & $-\frac{4}{\sqrt{\pi}} A^{-3}$ & $-\frac{48}{\sqrt{\pi}} A^{-1}$ & $\frac{192}{\sqrt{\pi}} \mathrm{A}$ & $\frac{256}{\sqrt{\pi}} A^{3}$ & $\frac{614.4}{\sqrt{\pi}} A^{5}$ \\
\hline 4 & $\frac{8}{\sqrt{\pi}} A^{-3}\left(1-\frac{6}{A^{2}}\right)$ & $\frac{32}{\pi} A^{-1}\left(3-\frac{10}{A^{2}}\right)$ & $-\frac{384}{\sqrt{\pi}} A\left(1+\frac{10}{A^{2}}\right)$ & $-\frac{512}{\sqrt{\pi}} A^{3}\left(1-\frac{30}{A^{2}}\right)$ & $\frac{1228.8}{\sqrt{\pi}} A^{5}\left(1-\frac{50}{3 A^{2}}\right)$ \\
\hline 5 & $-\frac{48}{\sqrt{\pi}} A^{-3}\left(1-\frac{4}{A^{2}}+\frac{39}{4}\right)$ & $-\frac{192}{\sqrt{\pi}} A^{-1}\left(3-\frac{20}{3 A^{2}}+\frac{42}{4}\right)$ & $\frac{768}{\sqrt{\pi}} A\left(3+\frac{20}{A^{2}}-\frac{70}{A^{4}}\right)$ & $\frac{3072}{\sqrt{\pi}} A^{3}\left(1-\frac{20}{A^{2}}-\frac{210}{A^{4}}\right)$ & $\frac{8192}{\sqrt{x}} A^{5}\left(0.9-\frac{10}{A^{2}}+\frac{315}{A^{4}}\right)$ \\
\hline
\end{tabular}

known, the melted thickness $\xi$ is obtained from Eq. (34b), $X_{0}$ from Eq. (24), $Q^{\prime}$ from Eqs. (14) and (24), and the temperature from Eq. (15a).

Two equivalent procedures for the evaluation of these constants will be outlined: the first consists in obtaining a numerical solution of Eqs. (27) and (40) directly, while the second requires first a reduction to a more convenient set of equations and variables. Before proceeding, it should be noted that the coefficients $I_{i i}$ can be written as:

for $i=1,2$ and $j=1$ see the entries in Table I

for $i=1$ and $j \neq 1$

$\frac{\pi^{1 / 2} I_{1 j}}{4^{j-1} A^{2 j-1}}$

$$
\begin{aligned}
= & \frac{(-1)^{i}(2 j-3)(2 j-3) ! !}{2^{i+1} A^{2 j-1}}\left[\pi^{3 / 2}-4 I(A)\right]-\frac{1}{2 A^{2}}+\left(1-\delta_{2 j}\right) \sum_{k=2}^{i-1} \frac{(-1)^{k}(2 j-3) ! !}{2^{k}(2 j-2 k-1) ! !} \\
& \cdot\left\{\frac{1}{A^{2 k}}-\left(1-\delta_{2 k}\right) \sum_{m=0}^{k-3}\left(1-\delta_{m, k-2}\right) \sum_{n=0}^{k-m-3} \frac{A^{-2(n+m+2)}}{(k-m-n-2) !}\right. \\
& \left.\cdot\left[\frac{1}{k-m-n-2}+\left(1-\delta_{3, k-m-n}\right) \sum_{i=0}^{k-m-n-4} \frac{1}{k-m-n-l-3}\right]\right\} \\
+ & \frac{(-1)^{i+1}}{2^{j-1}} \sum_{m=0}^{i-2}\left(1-\delta_{m, i-2}\right) \sum_{n=0}^{i-m-3} \frac{(2 j-3) ! ! A^{-2(n+m+2)}}{(2 j-2 m-2 n-5)(j-m-n-3) !} \\
& \cdot\left[\frac{2}{2 j-2 m-2 n-5}+\left(1-\delta_{n, i-m-3}\right) \sum_{l=0}^{i-m-n-4} \frac{1}{j-m-n-l-3}\right] \\
+ & \frac{1}{2} e^{A^{2}} \operatorname{Ei}\left(A^{2}\right)\left[1-\frac{(-1)^{i}(2 j-3) ! !}{2^{j-1} A^{2(j-1)}}+\left(1-\delta_{2 j}\right) \sum_{k=2}^{i-1} \frac{(-1)^{k}(2 j-3) ! !}{2^{k-1}(2 j-2 k-1) ! !} \frac{k-A^{2}-1}{A^{2 k}}\right]
\end{aligned}
$$


for $i=2$ and $j \neq 1$

$$
\begin{aligned}
& \frac{\pi^{1 / 2} I_{2 j}}{4^{j} A^{2 j-3}}=-\frac{1}{2}+\frac{(-1)^{i}(j-1)(2 j-3) ! !}{2^{j} A^{2 i-3}}\left[\pi^{3 / 2}-4 I(A)\right] \\
& +\frac{1}{2} e^{A^{2}} E i\left(A^{2}\right)\left[A^{2}-j+1-\left(1-\delta_{2 j}\right)(j-1)(2 j-3) ! ! \sum_{k=3}^{i} \frac{(-1)^{k} A^{-2(k-2)}}{2^{k-2}(2 j-2 k+1) ! !}\right] \\
& +\left(1-\delta_{2_{j}}\right) \frac{(j-1)(2 j-3) ! !}{2^{j-1}}\left\{( - 1 ) ^ { i - 1 } \sum _ { m = 0 } ^ { j - 3 } \left[\frac{2 A^{-2(m+1)}}{(2 j-2 m-5)^{2}(j-m-3) !}\right.\right. \\
& \left.+\frac{\left(1-\delta_{3, i-m}\right) A^{-2(m+1)}}{(2 j-2 m-5)(j-m-3) !} \sum_{n=0}^{i-m-4} \frac{1-m-n-3}{j-m}\right]+2^{i} \sum_{k=3}^{j} \frac{(-1)^{k}\left(1-\delta_{k 3}\right)}{2^{k}(2 j-2 k+1) ! !} \\
& \left.\quad \cdot \sum_{m=0}^{k-4} \frac{A^{-2(m+1)}}{(k-m-3)(k-m-3) !}\left[1+\left(1-\delta_{4, k-m}\right) \sum_{n=0}^{k-m-5} \frac{k-m-3}{k-m-n-4}\right]\right\}
\end{aligned}
$$

for $i \geq 3$

$$
\begin{aligned}
& \frac{\pi^{1 / 2} I_{i j}}{4^{i+i-2} A^{2 i-5}}=\sum_{n=1}^{i}(-1)^{n}\left(1-\delta_{i+j-1, n}\right) \alpha_{n}(i) \sum_{m=0}^{i-3} \frac{(i-3) ! A^{-2 m}}{(i-3-m) ! m !} \\
& \sum_{k=0}^{(i-n) H(i-2)+(i+i-n-2) H(1-j)} \frac{(2 i+2 j-2 n-3) ! !(i+m-n-k) !}{2^{k+1}(2 i+2 j-2 n-2 k-3) ! !} \\
& -H(j-3) D_{i j}\left\{\sum_{k=0}^{(i-3) H(i-i)+(j-3) H(i-j-1)} \frac{(-1)^{i-k}(i-3) ! A^{-2 k}}{k !(i-3-k) !(2 j-2 k-5)(j-k-3) !}\right. \\
& +\left[\frac{2 H(j-k-3)}{2 j-2 k-5}+\left(1-\delta_{3, i-k}\right) \sum_{m=1}^{i-k-3} \frac{H(j-k-m-2)}{j-k-m-2}\right] \\
& +\sum_{k=0}^{i-3} \frac{2^{i-k-3}}{(2 j-2 k-5) ! !}\left[H(i-k-4) \sum_{r=k+1}^{i-3} \frac{(i-3) !(r-k-1) !}{(i-3-r) ! r ! A^{2 r}}\right. \\
& \left.\left.+\sum_{r=0}^{k H(i-k-3)+(i-3) H(k-i+2)} \frac{\left(1-\delta_{k r}\right)(-1)^{k-r}(i-3):}{r !(k-r) !(i-3-r) ! A^{2 r}} \sum_{m=1}^{k-r} \frac{H(k-r-m+1)}{k-r-m+1}\right]\right\}
\end{aligned}
$$

where $I(A)$ is defined in Eq. (44) and where

$$
\begin{aligned}
\alpha_{n}(i) & =(-1)^{n-1} 4^{1-i} \gamma_{n}(i-1), \\
D_{i j} & =\sum_{n=1}^{i}(-1)^{n-1} \alpha_{n}(i) \frac{(2 i+2 j-2 n-1) ! !}{2^{i+\frac{i}{n-1}}(2 i+2 j-2 n-1)}, \\
\delta_{i i} & = \begin{cases}1, & i=j, \\
0, & i \neq j,\end{cases} \\
H(i) & = \begin{cases}0, & i<0, \\
1, & i \geq 0,\end{cases} \\
(2 n-1) ! ! & =1 \cdot 3 \cdot 5 \cdots(2 n-1)
\end{aligned}
$$

where $\gamma_{n}(i)$ was defined in Eq. (18).

These expressions are derived from (28) by successive integrations by parts in [23]. 
Explicit expressions for the coefficients in the first five columns and rows are shown in Table I. It may be verified that these coefficients enjoy the following properties:

1. The coefficients of the first two rows are expressible in terms of polynomials in $A$ (with positive powers except for $\left.I_{21}\right)$, of the tabulated functions $e^{A^{2}}$ and $\mathrm{Ei}\left(A^{2}\right)$, and of the integral

$$
I(A)=\frac{\pi^{3 / 2}}{4}-\frac{\pi^{1 / 2}}{4} \int_{0}^{1} \frac{\operatorname{erf}\left(A\left(\frac{1-Y}{Y}\right)^{1 / 2}\right)}{(Y(1-Y))^{1 / 2}} d Y \equiv \int_{0}^{\infty} e^{-u^{2}} \arctan \left(\frac{u}{A}\right) d u
$$

which represents a bounded monotone decreasing function of $A$ (see Fig. 2). Expressions for the coefficients $I_{1 i}$ and $I_{2 i}$ valid for large values of $A$ are collected in Table II and discussed further in Sec. 5 .

2. The coefficients in the rows beyond the second $(i \geq 3)$ are polynomials with positive and negative powers of $A$.

3. Note that $I_{i i} \neq I_{i i}$.

(a) First method of solution. The solution of Eqs. (27) can be formally represented as

$$
A_{i}=-C_{1} I_{i 1}^{-1}(A)+C_{2} I_{i 2}^{-1}(A)
$$

where $I_{i 1}^{-1}, I_{i 2}^{-1}$ are elements of the matrix $\left[I_{i j}\right]^{-1}$, inverse to $\left[I_{i j}\right]$. The coefficients $I_{i j}$ were calculated for several values of $A$, and the inverse coefficients $I_{i i}^{-1}$ were then obtained by numerical inversion of the matrix. In this work, matrix sizes from $3 \times 3$ to $9 \times 9$ were employed. The resulting values of $A_{i}$, in terms of $C_{1}$ and $C_{2}$ from Eq. (45), were then substituted into Eq. (40), which could be directly solved for $\left(C_{2} / C_{1}\right)$ for each particular value of $A$. Plots of $A$ against $\left(C_{1} / C_{2}\right)$ are shown in Fig. 3 ; two curves are given, using

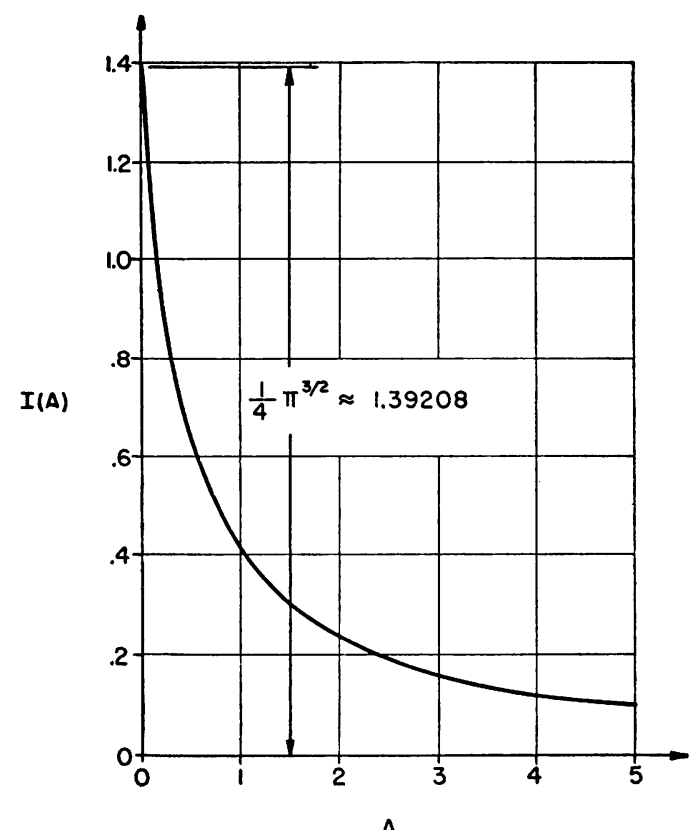

Fig. 2. Representation of $I(A)=\int_{0}^{\infty} e^{-u^{2}} \tan ^{-1}(u / A) d u$. 
TABLE II

Asymptotic expressions for coefficients $I_{i j}$ for $i=1,2, j=1,2, \ldots, 5$

\begin{tabular}{|c|c|c|c|c|c|}
\hline$i$ & 1 & 2 & 3 & 4 & 5 \\
\hline 1 & $\frac{\pi}{4}-\frac{1}{\sqrt{\pi}}\left(\frac{1}{3 A^{3}}-\frac{4}{3 A^{5}}+\cdots\right)$ & $\begin{array}{l}\frac{\pi}{2}-\frac{2}{\sqrt{\pi}}\left(\frac{2}{A}-\frac{2 ! 4}{3 A^{3}}\right. \\
\left.\frac{3 ! 6}{35^{5}}+\cdots\right)\end{array}$ & $\begin{array}{l}\frac{16}{\sqrt{\pi}} \mathrm{A}-9 \pi \\
+\frac{16}{\sqrt{\pi}}\left(\frac{4}{\mathrm{~A}}-\frac{6}{\mathrm{~A}^{3}}+\cdots\right)\end{array}$ & $\begin{array}{l}\frac{64}{\sqrt{\pi}}\left(\frac{A^{3}}{3}-4 A\right)+150 \pi \\
+\frac{4}{\sqrt{\pi}}\left(-\frac{213}{A}+\frac{1,87}{A^{3}}+\cdots\right)\end{array}$ & $\begin{array}{l}\frac{256}{\sqrt{\pi}}\left(\frac{A^{5}}{5}-\frac{4}{5^{3}}+18 A\right)-2940 \pi \\
+\frac{16}{\sqrt{\pi}}\left(\frac{1152}{A}-\frac{3200}{A^{3}}+\ldots\right)\end{array}$ \\
\hline 2 & $\begin{array}{l}-\frac{2}{\sqrt{\pi}} A^{-3}\left(1-\frac{2 !}{A^{2}}+\right. \\
\left.\frac{3 !}{A^{4}}+\cdots\right)\end{array}$ & $\begin{array}{l}4 \pi-\frac{4}{\sqrt{\pi}}\left(\frac{6}{A}-\frac{20}{3 A^{3}}+\right. \\
\left.\frac{84}{5 A^{5}}+\cdots\right)\end{array}$ & $\begin{array}{l}\frac{96}{\sqrt{\pi}} \mathrm{A}-48 \pi \\
+\frac{32}{j \pi}\left(\frac{10}{\mathrm{~A}}-\frac{14}{\mathrm{~A}^{3}}+\cdots\right)\end{array}$ & $\begin{array}{l}\frac{128}{\sqrt{\pi}}\left(A^{3}-10 A\right)+720 \pi \\
+\frac{128}{\sqrt{\pi}}\left(-\frac{42}{A}+\frac{72}{A}+\cdots\right)\end{array}$ & $\begin{array}{l}\frac{1024}{\sqrt{\pi}}\left(\frac{3}{10^{A}} \mathrm{~A}^{5}-\frac{5}{3^{3}}+21 A\right)-13440 \pi \\
+\frac{1024}{\sqrt{\pi}}\left(\frac{108}{A}-\frac{220}{A^{3}}+\cdots\right)\end{array}$ \\
\hline
\end{tabular}

either these variables or their reciprocals, so as to indicate clearly the behaviors near $A=C_{1} / C_{2}=0$ and near $A=C_{1} / C_{2}=\infty$.

Once the relationship between $A$ and $\left(C_{1} / C_{2}\right)$ is established, the values $\left(A_{i} / C_{1}\right)$ can be calculated from Eqs. (45). The solution is now complete.

Plots of $\left(A_{1} / C_{1}\right)$ and $\left(A_{2} / C_{1}\right)$ against $\left(C_{2} / C_{1}\right)$ are shown in Fig. 4 . It is of interest to note that the coefficients $A_{i}$ decrease rapidly with increasing $i$ (Fig. 5) and do not change
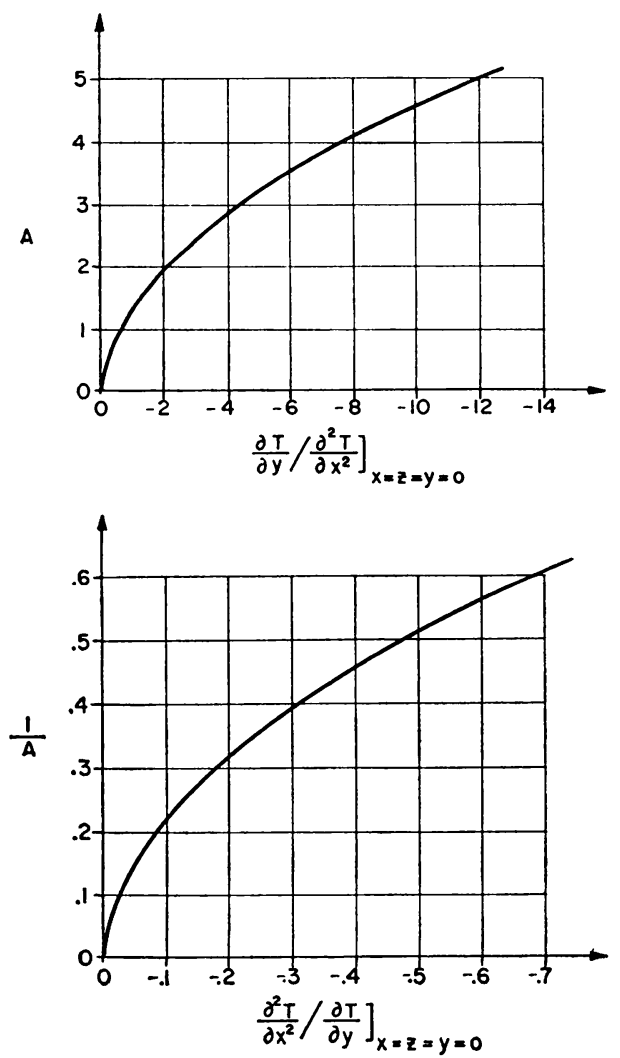

Fra. 3. Variation of $A$ and $1 / A$ with ratios of initial temperature derivatives. 


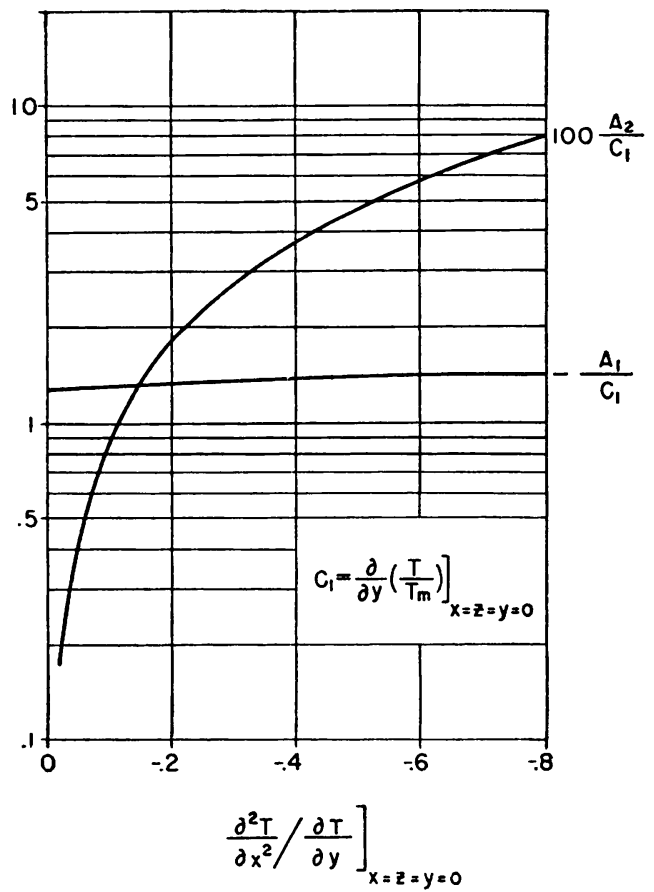

FIg. 4. Variation of $A_{1} / C_{1}$ and $A_{2} / C_{1}$ with the ratio of initial temperature derivatives.

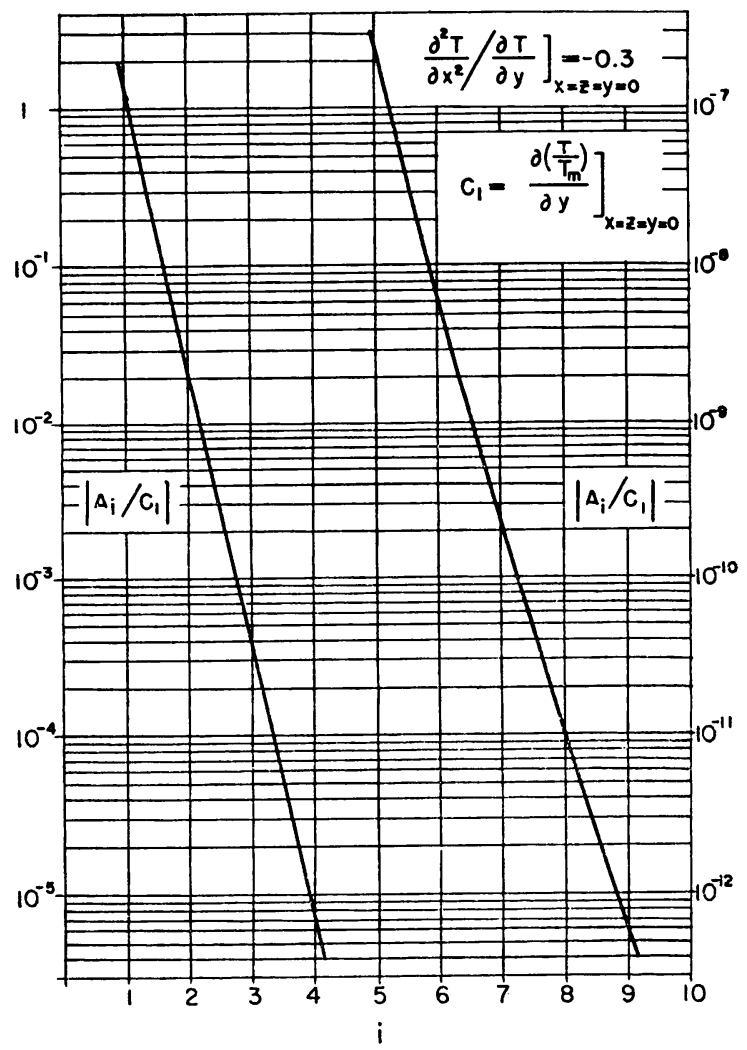

Fig. 5. Variation of coefficients $A_{i}$ for values of $i=1,2, \ldots \ldots, 9$. 
TABLE III

Coefficients $A_{i} / C_{1}$ resulting from increasing matrix sizes; $A=10, i=1,2,3$

\begin{tabular}{|c|c|c|c|c|}
\hline Matrix size & $A_{1} / C_{1}$ & $A_{2} / C_{1}$ & $\left(A_{3} / C_{1}\right) \times 10^{5}$ & \\
\hline 3 & -1.27737 & .002129 & .266 & \\
\hline 4 & -1.27697 & .001915 & .160 & \\
\hline 5 & -1.27680 & .001824 & .137 & \\
\hline 6 & -1.27670 & .001773 & .127 & $\partial(T)$ \\
\hline 7 & -1.27664 & .001741 & .121 & $C_{1}=\frac{d}{\sigma}\left(\frac{T}{T}\right)$ \\
\hline 8 & -1.27659 & .001718 & .117 & $X=0$ \\
\hline \multirow[t]{2}{*}{9} & -1.27656 & .001702 & .115 & $Z=0$ \\
\hline & & & & $y=0$ \\
\hline
\end{tabular}

radically with increasing size of matrix (Table III). Such numerical indications of convergence hold throughout the work presented here (e.g. Fig. 6 or Eq. (56)). A further check is provided by comparisons with the expressions valid for large values of $A$, developed in Sec. 5. In that section, some approximate results are also given.

(b) Second method of solution. Inspection of Table I reveals that the coefficients shown in the third row are

$$
I_{3 i}=\frac{3\left(4^{i}\right) A^{2 i-5}}{(2 j-5) \pi^{1 / 2}}
$$

These are proportional to successive terms of the series in Eq. (37); hence, the equation

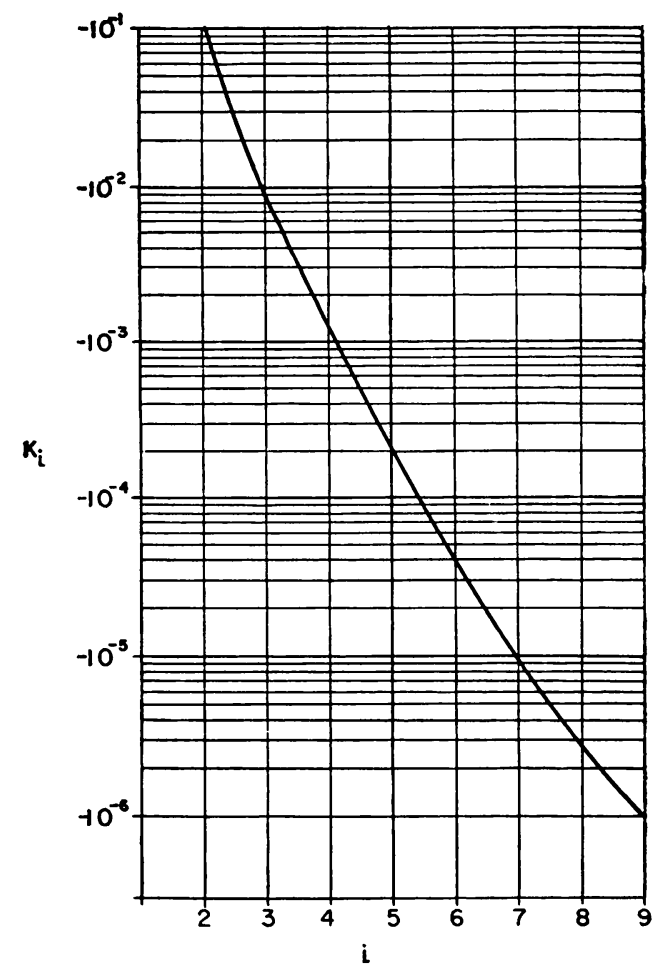

Fig. 6. Values of $K_{i}$ for $i=1,2, \cdots, 9$. 
corresponding to $i=3$ is identical with (37) and thus, as was to be shown, Eqs. (27) and (35) are not independent.

It can now further be noticed that the first term listed in Table $I$ for $I_{4 i}$ is equal to $\left(-2 I_{3 i}\right)$, while the first term of $I_{5 i}$ is $\left(12 I_{3 i}\right)$. Also, the ratio of the second term of $I_{5 i}$ to the second term of $I_{4 i}$ is $(-4)$ for all $j$. If it is assumed that similar relationships hold for all values of $i \geq 3$ and $j$, then linear combinations of the equations for $i \geq 3$ can be written so that each coefficient has only one term, namely the last term of each polynomial. Furthermore, coefficients pertaining to neighboring values of $j$ in each of the resulting equations are proportional to powers of $A$ differing by 2. It follows that Eqs. (27) for $i \geq 3$ can be reduced to

$$
\sum_{i=1}^{\infty} I_{i j}^{\prime} B_{i}=0, \quad i=3,4,5, \cdots
$$

where the new variables

$$
B_{i}=A^{2(j-1)} A_{i}
$$

have been introduced.

It is important to note that the coefficients $I_{i j}^{\prime}$ are independent of $A$. Thus a solution of Eqs. (47) will give $B_{i}$ for $i \geq 3$ as a linear combination of $B_{1}$ and $B_{2}$. However, Eq. (40) becomes, in terms of the new variables,

$$
\sum_{i=1}^{\infty} 4^{i-1} B_{i}=0
$$

and therefore, by combining (49) and (47), one can determine all $B_{i}$ coefficients for $i \geq 2$ in terms of $B_{1}$, namely

$$
B_{i}=K_{i} B_{1} .
$$

Clearly $K_{1}=1$ and $A_{1}=B_{1}$. This calculation was performed by the procedure just outlined and, alternatively, by calculating the values of $K_{i}$ (which again are independent of $A$ ) from the equation

$$
K_{i}=A^{2(i-1)} A_{i} / A_{1}
$$

on the basis of the coefficients $A_{i}$ obtained by the first method of solution for several values of $A$. Since it was found that, in this latter calculation, the coefficients $K_{i}$ were indeed independent of the particular value of $A$ used, it was concluded that the previous conjecture concerning the behavior of the $I_{i j}$ coefficients was correct. Values of $K_{i}$ are given in Fig. 6. The coefficients $A_{i}$ are obtainable immediately from this information since

$$
A_{i}=K_{i} A_{1} / A^{2(i-1)} \text {. }
$$

There now remains to obtain the values of $A_{1}$ and $A$ : these must be obtained from the equations corresponding to $i=1,2$ in Eqs. (27). These can now be written as

$$
A_{1} \sum_{i=1}^{\infty} K_{i} I_{1 j}(A)=-C_{1}, \quad A_{1} \sum_{i=1}^{\infty} K_{i} I_{2 j}(A)=+C_{2} .
$$

Thus the relation between $A$ and $\left(C_{2} / C_{1}\right)$ is

$$
\frac{C_{2}}{C_{1}}=-\frac{\sum_{i=1}^{\infty} K_{j} I_{2 j}(A)}{\sum_{j=1}^{\infty} K_{j} I_{1 j}(A)}
$$

which has already been plotted in Fig. 3. Similarly 


$$
\frac{A_{1}}{C_{1}}=-\frac{1}{\sum_{i=1}^{\infty} K_{i} I_{1 i}(A)}
$$

which leads to the plot of Fig. 4.

5. Summary of results and approximations; behavior for large A. The starting solution of the problem formulated in Sec. 2 may now be summarized as follows. The thickness of melted material is given by Eqs. (34b), (52) and Fig. 5, or

$$
\begin{aligned}
\xi(X, y)= & -m A_{1} y^{3 / 2} \sum_{i=1}^{\infty} \frac{4^{i-1}}{5-2 i} K_{i}\left[\frac{X}{X_{0}(y)}\right]^{2(i-1)} \\
=-\frac{m}{3} A_{1} y^{3 / 2}\left[1-1.600\left(\frac{X}{X_{0}}\right)^{2}+.431\left(\frac{X}{X_{0}}\right)^{4}+.078\left(\frac{X}{X_{0}}\right)^{6}\right. & \left.\quad+.033\left(\frac{X}{X_{0}}\right)^{8}+.019\left(\frac{X}{X_{0}}\right)^{10}+.013\left(\frac{X}{X_{0}}\right)^{12}+\cdots\right] .
\end{aligned}
$$

Of course, $\xi=0$ for $|X| \geq X_{0}(y)$, where the extent of the melted material along the surface is given by

$$
X_{0}(y)=A y^{1 / 2} .
$$

A plot of the melted thickness is shown in Fig. 7. The parameters $A$ and $A_{1}$ are given

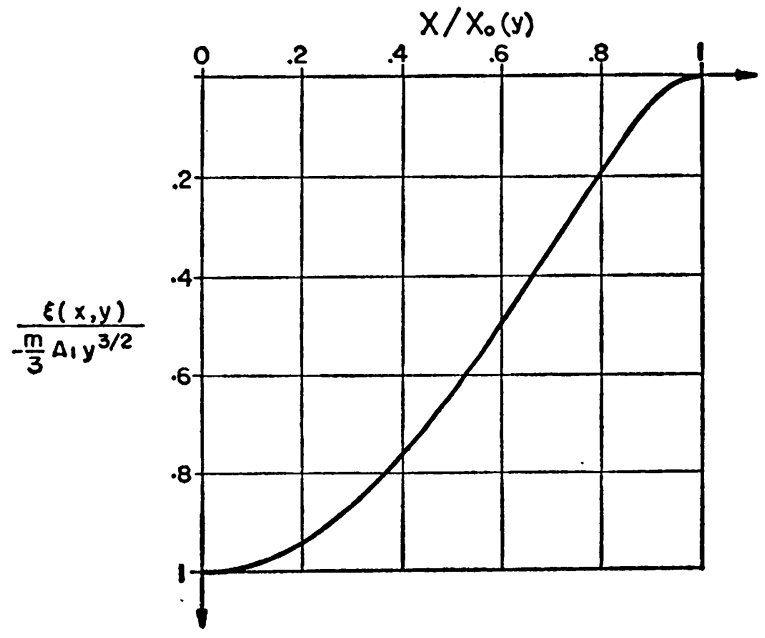

FIG. 7. Melt depth shape.

respectively in Figs. 3 and 4.

The fictitious heat input $Q^{\prime}$ is given by Eq. (32), or

$$
\begin{aligned}
\frac{Q^{\prime}(X, y)}{Q_{0}}= & -\frac{m}{2} \frac{\partial \xi}{\partial y}(X, y)=A_{1} y^{1 / 2} \sum_{i=1}^{\infty} 4^{i-1} K_{i}\left[\frac{X}{X_{0}(y)}\right]^{2(i-1)} \\
= & A_{1} y^{1 / 2}\left[1-.533\left(\frac{X}{X_{0}}\right)^{2}-.144\left(\frac{X}{X_{0}}\right)^{4}-.078\left(\frac{X}{X_{0}}\right)^{6}\right. \\
& \left.\quad-.054\left(\frac{X}{X_{0}}\right)^{8}-.044\left(\frac{X}{X_{0}}\right)^{10}-.039\left(\frac{X}{X_{0}}\right)^{12}-\cdots\right] .
\end{aligned}
$$

A plot of this equation is shown in Fig. 8 . 


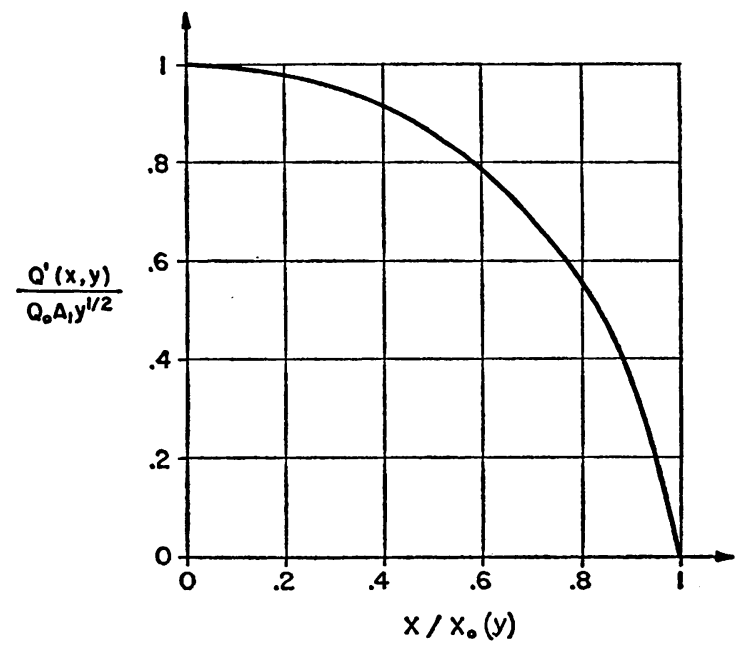

Fig. 8. Fictitious heat-input shape.

Some approximations to the above exact results can be obtained from the following considerations of the behavior for large values of $A$. Table II gives the coefficients of the first two $(i=1,2)$ of Eqs. (27), obtained by means of asymptotic expansions of the transcendental functions appearing there. Comparison of the terms in Table II with those of Eqs. (27) for $i \geq 3$, given in Table I, shows that all the coefficients of negative odd powers of $A$ vanish, so that the first two equations of (27) become, from Eqs. (42):

$$
\begin{gathered}
\pi \sum_{i=1}^{\infty}(-1)^{i} 2^{j-3}(2 j-3)(2 j-3) ! ! K_{j} A^{2(1-i)}=-C_{1} / A_{1}, \\
\pi \sum_{j=2}^{\infty}(-1)^{i} 2^{i}(j-1)(2 j-3) ! ! K_{i} A^{2(1-i)}=C_{2} / A_{1},
\end{gathered}
$$

which are valid only for $A \gg 1$. If the coefficients $K_{i}$ previously found are employed, these two equations can be solved simultaneously for the two unknowns $A$ and $\left(A_{1} / C_{1}\right)$ as functions of $\left(C_{1} / C_{2}\right)$. Thus

$$
A=4\left(-K_{2} \frac{C_{1}}{C_{2}}\right)^{1 / 2}+\frac{1}{4}\left(1+6 \frac{K_{3}}{K_{2}^{2}}\right) \sqrt{-K_{2} \frac{C_{2}}{C_{1}}}+O\left(\frac{C_{2}}{C_{1}}\right)^{3 / 2}
$$

or, approximately, keeping one term only,

$$
A \doteq 4\left(-K_{2} \frac{C_{1}}{C_{2}}\right)^{1 / 2} \doteq \sqrt{-2 \frac{\left(\partial T^{*} / \partial y\right)_{0,0,0}}{\left(\partial^{2} T^{*} / \partial X^{2}\right)_{0,0,0}}} .
$$

It may be verified that the latter expression actually fits the curve of Fig. 3 quite well for all values of $A^{10}$, provided that $K_{2}$ is guessed to be $\left(-\frac{1}{8}\right)$.

The corresponding expressions for $\left(A_{1} / C_{1}\right)$ is then obtained by substitution into the first of (59) as

$$
\frac{A_{1}}{C_{1}}=-\frac{4}{\pi}\left(1-\frac{2 K_{2}}{A^{2}}\right)+O\left(\frac{1}{A^{4}}\right)
$$

${ }^{10}$ The authors are indebted to the reviewer of this paper for pointing out to them the validity of the last of approximations (60a) for the parameter $A$ and of (62) for the shape of the melt line. 
or, with (60a) and independently of the value of $K_{2}$,

$$
A_{1} / C_{1} \doteq-(4 / \pi)\left(1+C_{2} / S C_{1}\right) \text {. }
$$

It may be noticed that this equation fits well the curve of Fig. 4 in the range plotted there; for larger values of $C_{2} / C_{1}$, larger errors arise.

It may furthermore be noticed by inspection of Fig. 7 that the equation

$$
\frac{\xi}{-\frac{m}{3} A_{1} y^{3 / 2}}=\left[1-\left(\frac{X}{X_{0}}\right)^{2}\right]^{3 / 2}
$$

is a good approximation to the shape of the melt line.

6. Discussion of the solution. The following remarks may be made concerning the solution presented in the preceding section:

1. The solution depends, when put in dimensionless form, upon two dimensionless parameters, namely $C_{1}=\partial\left(T / T_{m}\right) / \partial y$ and $C_{2}=-\partial^{2}\left(T / T_{m}\right) / \partial X^{2}$, both evaluated at $X=Z=y=0$.

2. Equation (56) indicates the remarkable fact that, for very short times after the start of melting, the shape of the melt depth is independent of the physical parameters of the problem. The magnitude of the melt depth of course does depend on these parameters; however, as Fig. 4 and Eq. (61a) show, it is insensitive to variations in $C_{2}$ and is roughly proportional to $C_{1}$.

3. Equation (60) and Fig. 3 show that, as $C_{2} \rightarrow 0, A \rightarrow \infty$ and therefore $X_{0} \rightarrow \infty$. This means that only the first term of the expansion in Eq. (56) is then significant for any finite value of $X$; hence in this case the solution reduces (with Eq. (61a)) to

$$
\xi(y)=\frac{4 m}{3 \pi} \frac{\partial\left(\frac{T}{T_{m}}\right)}{\partial y} y^{3 / 2},
$$

in exact agreement with the known starting solution to the analogous one-dimensional problem [9].

4. It is interesting to note that melting progresses much faster, for short times at least, along the surface of the body than into the interior. Indeed the distance traveled by the melt depth along the surface is initially proportional to $\left(t-t_{m}\right)^{1 / 2}$, while the penetration into the body is proportional to $\left(t-t_{m}\right)^{3 / 2}$. This implies that two-dimensional effects have a tendency to decrease with time. To obtain an experimental verification of the present results, a much more refined time scale would have to be used than in the corresponding one-dimensional problem. A meaningful experiment would have to be designed so that the ratio $\left(C_{2} / C_{1}\right)$ is large so as to emphasize the two-dimensional effect; at the same time $C_{1}$ should be adequately large to produce significant melting in a reasonable time.

5. The solution presented here has been restricted to the case in which Eqs. (21) and (33) hold; it reduces, as has been noted above, to the one-dimensional solution appropriate to these conditions, which, in particular gives $\xi \propto y^{3 / 2}$ as in Eq. (63). It may be conjectured that the behavior will also be similar, in the two-dimensional and one-dimensional cases, if the above restrictions are removed. If that is the case, then a finite jump in the applied heat input should lead to $\xi \propto y$, while an infinite jump (corresponding to a sudden finite jump in surface temperature) should lead to $\xi \propto y^{1 / 2}$.

6. It was mentioned in the Introduction that it was hoped that the present type of 
analysis would point the way to possible applications to the study of dendritic growth. Examination of published shapes of dendritic clusters ([14], [16], [17]) shows that much flatter growths are observed than would be expected from Fig. 7. Indeed, from some approximate calculations in [16], it is expected that the average height of the growth would occur at about $X / X_{0} \approx 0.9$ rather than at $X / X_{0} \approx 0.6$ as in Fig. 7 . That such a discrepancy should occur is not surprising, since the present solution does not really duplicate the conditions of dendritic formation. It is known ([14], for example) that dendrites only form in the presence of supercooling which would imply (according to the conjecture under item 5 above) that $\xi \propto y^{1 / 2}$. It may be pointed out, however, that a more "pointed" shape than that often assumed [14] would lead to more accurate comparisons with experimental data on velocity of growth propagation, and that the parabolic shape assumed in [15] for dendritic growth is not too dissimilar from that of Fig. 7.

\section{Appendix A}

Explicit form of Eqs. (20) for $i=1,2,3$ :

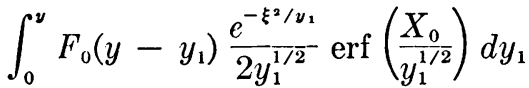

$$
\begin{aligned}
& +\int_{0}^{\nu} F_{1}\left(y-y_{1}\right) e^{-\xi^{2 / / y_{1}}} y_{1}^{1 / 2}\left[\operatorname{erf}\left(\frac{X_{0}}{y_{1}^{1 / 2}}\right)-\frac{2}{\pi^{1 / 2}} \frac{X_{0}}{y_{1}^{1 / 2}} e^{-X_{0} 2 / y_{1}}\right] d y_{1} \\
& +4 \int_{0}^{y} F_{2}\left(y-y_{1}\right) e^{-\xi^{2} / \nu_{1}} y_{1}^{3 / 2}\left[\frac{3}{2} \operatorname{erf}\left(\frac{X_{0}}{y_{1}^{1 / 2}}\right)-\frac{1}{\pi^{1 / 2}}\left(2 \frac{X_{0}^{3}}{y_{1}^{3 / 2}}-3 \frac{X_{0}}{y_{1}^{1 / 2}}\right) e^{-X_{0}{ }^{2} / y_{1}}\right] d y_{1}+\cdots \\
& =-y\left[\frac{\partial\left(\frac{T^{*}}{T_{m}}\right)}{\partial y}\right]_{0,0,0}+\cdots \\
& -2 \int_{0}^{\nu} F_{0}\left(y-y_{1}\right) \frac{e^{-\xi^{2} / y_{1}}}{\pi^{1 / 2} y_{1}^{2}} X_{0} e^{-X_{0}^{2} / y_{1}} d y_{1} \\
& +4 \int_{0}^{\nu} F_{1}\left(y-y_{1}\right) e^{-\xi^{2} / \nu_{1}}\left[\frac{1}{y_{1}^{1 / 2} / 2} \operatorname{erf}\left(\frac{X_{0}}{y_{1}^{1 / 2}}\right)-\frac{2}{\pi^{1 / 2}}\left(\frac{X_{0}^{3}}{y_{1}^{2}}+\frac{X_{0}}{y_{1}}\right) e^{-X_{0}^{2} / \nu_{1}}\right] d y_{1} \\
& +16 \int_{0}^{y} F_{2}\left(y-y_{1}\right) e^{-\xi^{2} / y_{1}}\left[3 y_{1}^{1 / 2} \operatorname{erf}\left(\frac{X_{0}}{y_{1}^{1 / 2}}\right)-\frac{2}{\pi^{1 / 2}}\left(2 \frac{X_{0}^{3}}{y_{1}}+3 X_{0}+\frac{X_{0}^{5}}{y_{1}^{2}}\right) e^{-X_{0}{ }^{2} / y_{1}}\right] d y_{1} \\
& +\cdots \\
& =-\left[\frac{\partial^{2}\left(\frac{T^{*}}{T_{m}}\right)}{\partial X^{2}}\right]_{0,0,0}+\cdots \\
& \frac{4}{\pi^{1 / 2}} \int_{0}^{\nu} F_{0}\left(y-y_{1}\right) \frac{e^{-\xi^{2} / y_{1}}}{y_{1}^{3}}\left(3-2 \frac{X_{0}^{2}}{y_{1}}\right) X_{0} e^{-X_{0} 2 / y_{1}} d y_{1} \\
& +\frac{16}{\pi^{1 / 2}} \int_{0}^{y} F_{1}\left(y-y_{1}\right) \frac{e^{-\xi^{2} / y_{1}}}{y_{1}^{2}}\left(\frac{X_{0}^{3}}{y_{1}}-2 \frac{X_{0}^{5}}{y_{1}^{2}}\right) e^{-X_{0}{ }^{2} / y_{1}} d y_{1} \\
& +32 \int_{0}^{y} F_{2}\left(y-y_{1}\right) \frac{e^{-\xi^{2} / y_{1}}}{y_{1}}\left[6 y_{1}^{1 / 2} \operatorname{erf}\left(\frac{X_{0}}{y_{1}^{1 / 2}}\right)\right. \\
& \left.-\frac{2}{\pi^{1 / 2}}\left(2 \frac{X_{0}^{7}}{y_{1}^{3}}+\frac{X_{0}^{5}}{y_{1}^{2}}+4 \frac{X_{0}^{3}}{y_{1}}+6 X_{0}\right) e^{-X_{0^{2} / y_{1}}}\right] d y_{1}+\cdots \\
& =-\left[\frac{\partial^{4}\left(\frac{T^{*}}{T_{m}}\right)}{\partial X^{4}}\right]_{0,0,0}+\cdots
\end{aligned}
$$


Appendix B

Reduction ${ }^{11}$ of the expression $D_{i i}$ for $j \leq i$ :

Expressions (18) and (43) render $D_{i j}$ in the form

$$
\begin{aligned}
D_{i j} & =-\frac{(-1)^{i}(2 i-2) !}{2^{2 i+j-3}} \sum_{k=0}^{i-1} \frac{(-1)^{k}(2 j+2 k-1) ! !}{(i-k-1) !(2 j+2 k-1)(2 k-1) ! ! k !} \\
& =-\frac{(-1)^{i}(2 i-2) !}{2^{2 i+i-3}} \sum_{k=0}^{i-1} \frac{(-1)^{k}}{(i-k-1) ! k !}\left[(2 k)^{i-1}+b_{2} k^{i-2}+b_{3} k^{i-3}+\cdots+b_{j}\right]
\end{aligned}
$$

where $k=i-n$ and

$$
(2 k) !=2^{k}(2 k-1) ! ! k !
$$

The definition of the quantity

$$
D_{i j}^{\prime}(x)=-\frac{(-1)^{i}(2 i-2) !}{2^{2 i+j-3}} \sum_{k=0}^{i-1} \frac{(-1)^{k}(2 j+2 k-1) ! ! x^{k}}{(i-k-1) !(2 j+2 k-1)(2 k-1) ! ! k !}
$$

is such that

$$
D_{i i}=D_{i i}^{\prime}(1)
$$

It will now be shown that

$$
D_{i j}^{\prime}(x)=-\frac{(-1)^{i}(2 i-2) !}{2^{2 i+j-3}} \sum_{m=0}^{i-1} a_{i-m} x^{m}(1-x)^{i-m-1}
$$

so that only positive powers of $(1-x)$ appear for $j<i$ and thus $D_{i j}^{\prime}(1)=0$. Hence $D_{i i}=0$ for $j<i$. On the other hand if $j=i$, only the term for $m=j-1$ will be different from 0 at $x=1$ and then

$$
D_{i i}=4^{1-i}(2 i-2) !
$$

where $a_{1}$ will be shown from the last of Eqs. (B13) to be

$$
a_{1}=(-1)^{i-1} 2^{i-1} /(i-j) !
$$

There remains to prove (B5), or, in other words, that $a_{m}$ exist.

For $i>j=1$, clearly

$$
\begin{aligned}
D_{i 1}^{\prime}(x) & =-\frac{(-1)^{i}(2 i-2) !}{2^{2 i-2}} \sum_{k=0}^{i-1} \frac{(-1)^{k}}{(i-k-1) ! k !} x^{k} \\
& =-\frac{(-1)^{i}(2 i-2) !}{2^{2 i-2}(i-1) !}(1-x)^{i-1} .
\end{aligned}
$$

For larger values of $j<i$, we proceed as follows. Using the binomial expansion

$$
\begin{aligned}
x^{m}(1-x)^{i-m-1} & =\sum_{r=0}^{i-m-1} \frac{(-1)^{r}(i-m-1) !}{(i-m-r-1) ! r !} x^{r+m} \\
& =\sum_{k=0}^{i-1} \frac{(-1)^{k-m}(i-m-1) !}{(i-k-1) ! k !} \frac{(k+1)(k) \cdots(k-m+1)}{(k+1)} x^{k}
\end{aligned}
$$

\footnotetext{
${ }^{11}$ The authors are indebted to Sara R. Boley for the proofs comprising the whole of Appendix B.
} 
where $k=r+m$, the following linear combination of functions is formed:

$$
\begin{aligned}
D_{i j}^{\prime}(x) & =-\frac{(-1)^{i}(2 i-2) !}{2^{2 i+j-3}} \sum_{m=0}^{i-1} a_{j-m} x^{m}(1-x)^{i-m-1} \\
& =-\frac{(-1)^{i}(2 i-2) !}{2^{2 i+i-3}} \sum_{k=0}^{i-1} \frac{(-1)^{k} x^{k}}{(i-k-1) ! k !} P\left(i, j, k, a_{1}, a_{2}, \cdots, a_{i-1}\right)
\end{aligned}
$$

where $b_{n, j-}$ is the coefficient of the sth power of $k$ in the polynomial

$$
k(k-1)(k-2) \cdots(k-j+n+1)
$$

so that

$$
\begin{aligned}
P\left(i, j, k, a_{1}, a_{2}, \cdots, a_{j-1}\right) & \\
= & (i-1) ! a_{i}+\sum_{s=1}^{j-1} k^{*} \sum_{n=1}^{j-i}(-1)^{i-n}(i-j+n-1) ! a_{n} b_{n, i-} .
\end{aligned}
$$

Equating coefficients of like powers of $x$ in (B3) and (B10), we obtain a system of equations from which we get $a_{n}$, namely

$$
\begin{aligned}
(i-1) ! a_{i} & =b_{i} \\
\sum_{n=1}^{i-i}(-1)^{i-n}(i-j+n-1) ! a_{n} b_{n, j-s} & =b_{i-s}, \quad \text { for } \quad 1 \leq s \leq j-1 .
\end{aligned}
$$

Clearly this system is triangular; further, the main-diagonal coefficients are not 0 and therefore a solution exists. In particular for $j=i, a_{1}=(-1)^{i-1} 2^{i-1}$ and hence

$$
D_{i i}=4^{1-i}(2 i-2) \text { ! for } j=i \text { and } D_{i i}=0 \text { for } j<i \text {. }
$$

\section{ReFERENCeS}

1. G. Lamé and B. P. E. Clapeyron, Memoire sur la solidification par refroidissement d'un globe liquide, Ann. Chimie Phys. 47, 250-256 (1831)

2. F. Neumann, Unpublished lectures (1835-40?) reported by H. Weber in Die partiellen DifferentialGleichungen der mathematischen Physik, nach Riemann's Verlesungen, Braunwshweig, 4th ed., Vol. II, 1901, pp. 118-122

3. J. Stefan, Ueber einige Probleme der Theorie des Wärmeleitung, Sitzungsberichte Kais. Akad. Wissenschaften, Vienna, 98, part II, pp. 473-484, 1889

4. M. Brillouin, Sur quelques problèmes non résolus de la physique mathématique classique: propagation de la fusion, Ann. Inst. H. Poincaré 1, 285 (1931)

5. D. L. Sikarskie and B. A. Boley, The solution of a class of two-dimensional melting and solidification problems, Int. J. of Solids and Structures 1(2), 207-234 (April 1965) and Technical Report No. 25, Contract No. Nonr 266(20), Department of Civil Engineering and Engineering Mechanics, Columbia University, May 1964

6. B. A. Boley, A method of heat-conduction analysis of melting and solidifying slabs, J. Math. Phys. 40 (4), 300-313 (1961)

7. B. A. Boley, "The analysis of problems of heat conduction and melting," High temperature structures and materials: Proc. of Symp. on Naval Structural Mechanics, Pergamon Press, Oxford, 1964, pp. 260-315

8. J. C. Muehlbauer and J. E. Sunderland, Heat conduction with freezing or melting, Appl. Mech. Rev. 18 (12), 951-959 (1965)

9. B. A. Boley, A general starting solution for melting and solidifying slabs, Int. J. Eng. Sci. 6, 89-111 (1968)

10. Ting-Shu Wu and B. A. Boley, Bounds in melting problems with arbitrary rates of liquid removal, SIAM J. Appl. Math. 14(2) (1966) 
11. B. A. Boley, Upper and lower bounds in problems of melting or solidifying slabs, Quart. J. Mech. Appl. Math. 7, 25:3-269 (1964)

12. S. J. Citron, On the conduction of heat in a melting slab, Proc. of the IVth U. S. Cong. of Applied Mechanics, A. S. M. E., June 1962, pp. 1221-1227

13. E. Friedman and B. A. Boley, Design estimates of stresses and deformations in melting plates, Technical Report No. 29, Contract No. Nonr 4259(07), Department of Civil Engineering and Engineering Mechanics, Columbia University, June 1965

14. B. Chalmers, Principles of solidification, Wiley, New York, 1961

15. G. Horvay and J. Cahn, Dendritic and spheroidal growth, Acta Metallurgica 9, 695-705 (1961)

16. W. A. Tiller, "Alloy Crystal Growth," Growth and Perfection of Crystals, Proc. of an Int. Conf. on Crystal Growth, General Electric Company, 1958

17. W. A. Tiller, Grain size control during ingot solidification, Part II: Columnar-equiaxed transition, Trans. of the Metallurgical Soc. of A. I. M. E. 224, 448-459 (1962)

18. B. A. Boley and J. H. Weiner, Theory of thermal stresses, Wiley, New York, 1960

19. H. S. Carslaw and J. C. Jaeger, Conduction of heat in solids, 2nd ed., Clarendon Press, Oxford, 1959

20. B. A. Boley, Estimate of errors in approximate temperature and thermal stress calculations, Proc. of the Eleventh Int. Cong. of Applied Mechanics, Munich, 1964, pp. 586-596

21. N. N. Lebedev, Spccial functions and their applications, Prentice-Hall, Englewood Cliffs, N. J., 1965, pp. 60

22. E. Goursat, Cours d'analyse mathematique, fifth ed., Vol. 3, Chapter 29, Gauthier-Villars, Paris, 1942

23. H. P. Yagoda, The starting solution for two-dimensional heat conduction problems with change of phase, Dissertation for the Eng. Sc. D. degree, Department of Civil Engineering and Engineering Mechanics, Columbia University, 1967 